\title{
A New Approach to Automatic Species Identification Using Biological Data Mining
}

\author{
Seetharam Narasimhan, Shreyas Sen and Amit Konar \\ Department of Electronics \& Telecommunication Engineering \\ Jadavpur University, Kolkata - 700032, India.
}

\begin{abstract}
The paper aims at designing a scheme for automatic identification of a species from its genome sequence. A set of 64 three-tuple keywords is first generated using the four types of bases: A, T, C, and G. These keywords are searched on $\mathrm{N}$ randomly sampled genome sequences, each of a given length (10,000 elements) and the frequency count for each of the $43=64$ keywords is performed to obtain a DNA-descriptor for each sample. Principal Component analysis is then employed on the DNA-descriptors for $\mathrm{N}$ sampled instances. The principal component analysis yields a unique feature descriptor for identifying the species from its genome sequence. The variance of the descriptors for a given genome sequence being negligible, the proposed scheme finds extensive applications in automatic species identification. Next, a computational map is trained by the Self-Organizing Feature Map algorithm using the DNA-descriptors from different species as the training inputs. The map is shown to provide an easier technique for recognition and classification of a species based on its genomic data.
\end{abstract}

\section{KEYWORDS}

biological data mining, species identification, DNA descriptors, principal components analysis, self-organizing feature map, feature descriptors

Reprint requests to: Seetharam Narasimhan, Department of Electronics and Telecommunication Engineering, Jadavpur University, Kolkata- 700032, India. e-mail: seetharam@gmail.com 


\section{INTRODUCTION}

Genomic data mining and knowledge extraction is an important problem in bioinformatics. The identification of a species from its genomic database is a challenging task. We propose to explore a new approach to extract genomic features of a species from its genome sequence.

Significant progress on DNA-string matching has been reported in the current literature on Bio-informatics. The most popular web-based tool is BLAST. Among the well-known techniques of DNA-string matching are the Smith-Waterman algorithm (Smith \& Waterman, 1981; Waterman \& Eggert, 1987) for local alignment, the Needleman-Wunsch algorithm (Needleman \& Wunsch, 1970) for global alignment, Hidden Markov's model, matrix model, evolutionary algorithms for multiple sequence alignment (Cai et al, 2000) etc. These works, though extremely valuable, have their limitations. The demerits include the use of complicated matrix algebra and dynamic programming, and the results of sequence matching are not free from pre-calculated threshold values. Noteworthily, none of the above-mentioned methods can be directly employed to identify the species from the structural signature of the genomes.

There is a dearth of rapid and parsimonious procedures that may be somewhat approximate in nature yet useful in producing quick and significant results. The idea is to make the analysis of large DNA sequences easier by statistically summarizing the data using dimensional reduction and clustering techniques, while capturing some of the fundamental structural information contained in the sequence data to help classify different species on the basis of their genomic data alone.

As the work entails processing huge amounts of incomplete or ambiguous data, the learning ability of artificial neural networks (ANNs) is utilized in this direction. The learning capabilities of ANNs, typically in data-rich environments, come in handy when discovering regularities from large datasets. There exist different ANN-based learning and rule mining strategies, with applications to the biological domain (Haykin, 1991).

First, we take into account frequency counts of 64 three-lettered primitive DNA attributes in randomly selected samples of the genome sequences of different species (e.g., the bacterium Escherichia coli ( $E$. coli) (Blattner et al, 1997), Drosophila melanogaster (Adams et al, 2000), Saccharomyces 
cerevisiae (yeast) (Cherry, et al, 1997), Mus musculus (mouse) and Homo sapiens (human beings)).

Second, to reduce the data dimension of extracted features (here, frequency count), principal component analysis (PCA) is employed on the randomly selected samples of genome sequence. The variance of the extracted feature vectors being extremely small for any randomly selected input sequence, the accuracy of the results in identifying the species is very high.

Third, clustering techniques are adopted on the frequency count data of three species: E. coli, Yeast and Mouse. The SOFM algorithm is adopted for this purpose. Maps of different dimensions are constructed and analyzed on the basis of their efficiency in clustering the extracted features from genomic data of different species.

\section{SELECTION OF DNA-DESCRIPTORS}

There are only 4 letters in a DNA-string; naturally, the substrings could be one lettered, two lettered, three lettered, or four lettered. Thus, the number of possible combinations in each case is $4,4^{2}, 4^{3}$, or $4^{4}$. Consequently, the total number of substrings would be $4+4^{2}+4^{3}+4^{4}$, which indeed is very large. To keep the search time optimum and moderately large search keys, we considered 3-lettered search keys only. Thus, we have $4^{3}=64$ search keys. Typical three-lettered search keys are AAA, AAC, AAG, AAT, ACA........ TTT. These 64 search keys thus generate a $(1 \times 64)$ frequency count vector, whose each component denotes population of one of the 64 sub-strings or keys in a sample of the genomic data of a species. The $(1 \times 64)$ vector is a DNA-descriptor of a given species. The DNA-descriptors are computed from different samples of a species and for different species as well.

\section{CREATION OF FEATURE DESCRIPTORS}

The methodology of employing PCA (Smith, L. 1., 2002) to the given problem is outlined below:

INPUT: A set of N DNA-descriptor vectors $(1 \times 64)$ representing the frequency counts of 64 three-tuple keywords. 
OUTPUT: A minimal feature descriptor vector sufficient to describe the problem without any significant loss in data.

i) Normalization: Let the $1^{\text {th }}(1 \times 64)$ input vector be denoted by

$$
\alpha_{i}=\left[\begin{array}{llll}
a_{i 1} & a_{i 2} & \ldots . . . & a_{264}
\end{array}\right]
$$

To get the vector normalized we use the following transformation:

$$
\text { aik } \leftarrow \frac{a i k}{\sum_{j=1}^{64} a_{i j}}
$$

ii) Mean adjusted data: To get the data adjusted around zero mean, we use the formula:

$$
a_{i k} \leftarrow a_{i k}-\overline{a_{i}} \text { where } \overline{a_{i}}=\text { mean of the } \mathrm{i}^{\text {th }} \text { vector }=\frac{1}{64} \sum_{j-1}^{64} a_{i v}
$$

The matrix $(\mathrm{N} \times 64)$ so obtained is called the Data Adjust

$$
=\left(\begin{array}{ccc}
a_{11} & \cdots & a_{164} \\
\vdots & \ddots & \vdots \\
a_{N 1} & \cdots & a_{N 64}
\end{array}\right)
$$

ii) Evaluation of the covariance matrix: The covariance between any two vectors $a_{i}$ and $a_{j}$ is obtained by the following formula:

$$
\operatorname{cov}\left(a_{i}, a_{j}\right)=c_{i j}=\frac{\sum_{k=1}^{6+}\left(a_{i k}-\bar{a}\right)\left(a_{j k}-\overline{a_{j}}\right)}{(n-1)}
$$

Covariance matrix $\mathrm{C}$ for the $\mathrm{N}$ different $(1 \times 64)$ vectors is represented as follows:

where $\mathrm{C}$ is an $\mathrm{N} \times \mathrm{N}$ matrix.

$$
C=\left(\begin{array}{ccc}
c_{11} & \cdots & \tilde{v}_{1 N} \\
\vdots & \ddots & \vdots \\
c_{N 1} & \cdots & c_{N N}
\end{array}\right)
$$

iv) Eigenvalue Evaluation: From the roots of the equation $|C-\lambda I|=0$, the eigenvalues of the covariance matrix $\mathrm{C}$ are obtained. There would be $\mathrm{N}$ 
eigenvalues of matrix $C$, and corresponding to each eigenvalue there would be eigenvectors each of dimension $\mathrm{N} \times 1$.

v) Principal Component Evaluation: The eigenvalues are not the same. In fact, it turns out that the eigenvector corresponding to the highest eigenvalue is the Principal Component $(\mathrm{N} \times 1)$ of the data set. Therefore

$$
\text { Principal Component }=\left[\begin{array}{c}
p_{1} \\
p_{3} \\
\vdots \\
p_{N}
\end{array}\right] \text { where } \quad \lambda_{\text {large }}>\lambda_{i} \text { for } 1 \leq t \leq
$$

vi) Projection of Data Adjust along the Principal Component: Now, to get the feature descriptor, the following formula is applied:

\section{Feature Descriptor $=$ Principal Component ${ }^{T} \times$ Data Adjust}

where Principal Component ${ }^{\top}(1 \times N)$ is the transpose of the Principal Component vector. Thus, we get a Feature Descriptor vector of dimension $1 \times$ 64 corresponding to $\mathrm{N}$ samples of the genome sequence database of the particular species.

vii) Computing the Mean Feature Descriptor: We calculate $M$ such feature descriptors from different random samples and then calculate the mean of these vectors and the variance vector (both $1 \times 64$ ) as well.

\section{GEOMETRIC REPRESENTATION OF FEATURE DESCRIPTOR}

The Feature Descriptor Diagrams for different species are described here. We could represent the feature descriptors using bar diagrams, pie-charts or any other standard representation. However, using the polar plot we get figures that are compact yet distinct representations of the mean feature descriptor. As mentioned earlier, the mean feature descriptor is a $1 \times 64$ vector. So to construct these diagrams $360^{\circ}$ is divided into 64 equal parts, corresponding to 64 keywords. Plotting it in polar $(r, \theta)$ co-ordinates with $r$ as the values of the mean feature descriptor vector and $\theta$ as these angles we get the feature descriptor diagrams. The feature descriptor diagrams are distinctly 
S. Narasimhan, S. Sen

and Amit Konar

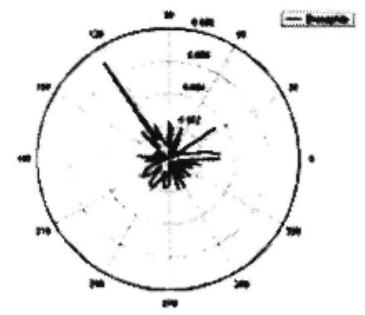

Fi. 1.1: Feature Descriptor Diagram

for Drosoptith

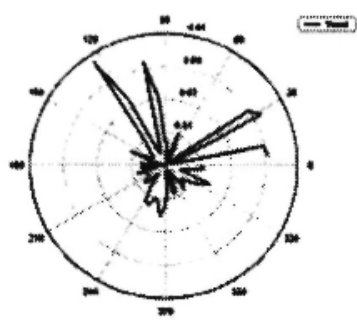
DE. 13: Feature Descriplor Diagrem for
Yens
Journal of Intelligent Systems

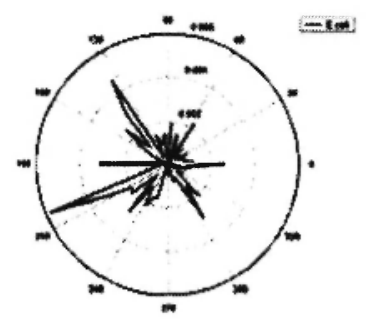

Fi. 1.2: Fenture Descriptor Dingrum for E. cols

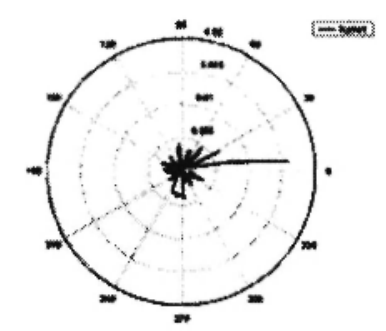

It- 1.4: Feature Descriplor Diagram for Humen chromosome spl1-433k2.

Fig. 1: Feature Descriptor Diagrams for Drosophila, E. coli, Yeast, and a Human chromosome

different from species to species. If we closely observe Figure 1.1, which contains the diagrammatic representation of the mean feature descriptor vector for Drosophila, we can see some distinct peaks with a prominent one at around $125^{\circ}$. In contrast, Figure 1.2 drawn for $E$. coli has its highest peaks near $125^{\circ}$ and $200^{\circ}$ and smaller ones around $140^{\circ}, 180^{\circ}$ and 300 . Similar distinctions are clearly visible from the other diagrams as well. So we can readily detect new species and identify known species by comparing their feature descriptor diagrams.

The DNA-descriptors obtained from different samples of the same species contain wide disparities. Hence, their diagrammatic representations alone cannot represent the species. Yet, the Feature Descriptors obtained after processing a different set of DNA-descriptors are unique and present absolutely no significant disparities. Hence, the Feature Descriptor Diagrams can be used as the unique representation of the genomic characteristics of the different species. 
We have plotted below the Feature Descriptors of different species obtained from their mitochondrial genomes. On seeing these diagrams, we can correctly conclude that the species Human, Gorilla, Chimpanzee and Pygmy Chimpanzee have many similarities in their genome characteristics which can be translated to a similarity in their biological characteristics.

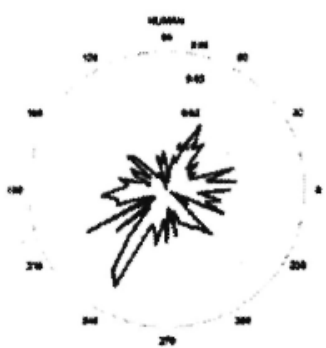

Fig. 2.1: Feature Descriptor Diagram for Homan

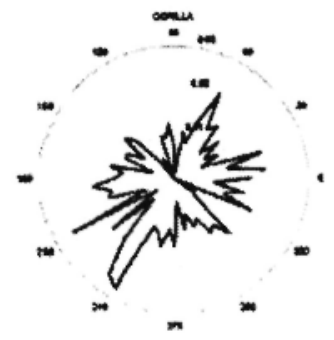

Fig. 2.3: Festure Descriptor Diagram for Gorilts

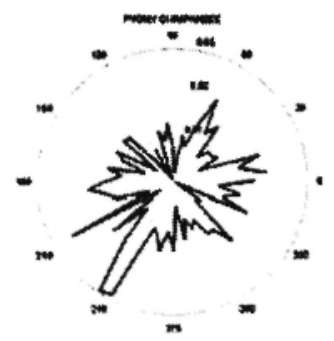

Fig. 2.5: Feabure Descriptor Disgram for Pyzmy Chrmpanzes

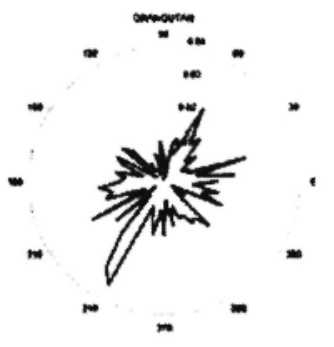

Fiz. 2.2: Feature Descriptor Dingram fox Orang-uten

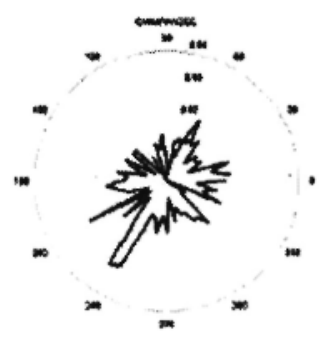

Fig. 24: Feature Descruptor Dingram for Chimpenzese

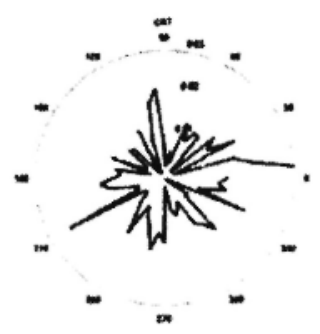

Fig. 2.6: Feature Decciptor Diagram for Cet

Fig. 2: Feature Descriptor diagrams for species obtained from their mitochondrial genomes 
However, the diagrams show quite clearly that these species have some distinctions in their genomic characteristics. On the other hand, the species like Cat and Anopheles mosquitoes, which have different characteristics, are found to have vividly distinct Feature Descriptor diagrams.

However, the cumbersome task remains of applying the abovementioned process to the genomic data of all the species to get figures corresponding to all the species. As an easier approach to automatic species identification we present in the next section a topological clustering method which will give us a single feature map whose different portions contain mappings from the extracted features of different species.

\section{TOPOLOGICAL CLUSTERING OF DNA-DESCRIPTORS BY SOFM}

In this section, we attempt to map the DNA-descriptors onto a $2 \mathrm{D}$ array of neurons by the well-known Self-organizing Feature Map algorithm. Our main interest is to note whether DNA-descriptors of the same species occupy neighborhood neuronal positions and species having close resemblances in their DNA structure form neighborhood clusters.

To verify the above, we considered 36 vectors of each of the following 3 species: Mouse, Yeast, and E. coli. Naturally, we have $36 \times 3=108$ vectors to be mapped onto the $2 \mathrm{D}$ array of size $(\mathrm{k} \times \mathrm{k})$. To perform the experiment, we considered $(6 \times 6)$ dimensional space for the $2 \mathrm{D}$ array of neurons. Later the maps were created for different dimensions ranging from 4 to 11 . Training principles are outlined below.

\subsection{Training the Map}

INPUT: A set of 108 DNA-descriptor vectors each of size $(1 \times 64)$ obtained from 36 samples from each of the abovementioned 3 species. The vectors are in normalized form.

OUTPUT: Clustering of the DNA-descriptors over a 2-D array of neurons.

Normalization: Let the $i^{\text {th }}(1 \times 64)$ input vector be denoted by

$$
a i=\left[\begin{array}{llll}
a i 1 & a i 2 & \ldots & a i 64
\end{array}\right]
$$

To get the vector normalized we use the following transformation: 


$$
a_{i k} \leftarrow \frac{a_{i k}}{\sum_{j-1}^{64} a_{i j}}
$$

Creation of Neuron Field: The neuron field of dimension $(k \times k)$ is constructed. Each neuron has a weight vector of $(1 \times 64)$ dimension. Initially, $k$ is chosen as 6 and later the process was repeated for different values ranging from 4 to 11 .

Initialization: All the $\mathrm{k}^{2}$ neurons are initialized with random values ranging between 0 and 1 . While initializing special care should be exercised to ensure that two neurons should not have identical weight vectors.

Choosing the value of learning rate constant $\boldsymbol{\eta}$ : In the beginning, we keep the value of $\eta$ (eta), the learning constant, high (0.9) and gradually decrease it with each epoch until it reaches a very small value and thereafter $\eta$ was kept constant at 0.005 . The equation which governs the decay of $\eta$ is given as:

$$
\eta=0.9 \times\left(1-\frac{e p o c h}{\tau}\right) \text { for epoch }<\tau
$$

where $\tau$ is a constant less than maximum value of epoch.

Choosing the size of neighborhood: Initially the neighborhood includes the entire neuronal space and is gradually decreased until it finally contains only the nearest neighbors of the winning neuron. Here the SOFM algorithm uses a neighborhood function that is convex, so as to avoid the occurrence of metastable states (Erwin, et al, 1992), which represent topological defects in the configuration of the feature map.

\subsection{Phases of Training Process}

We may decompose the adaptation of the synaptic weights in the network into two phases (Kohonen T., 1982), (Kohonen T., 1997):

a) Self-organizing or Ordering Phase: It is during this first phase of the adaptive process that the topological ordering of the weight vectors takes place. In this phase, $\eta$ is provided with a high value $(0.9)$ and the neighborhood is defined large enough so that all the neurons are trained initially when any neuron wins for a particular data input. The neighborhood size also decreases in the subsequent epochs. 
b) Convergence Phase: The second phase of the adaptive process is needed to fine tune the feature map and therefore provide an accurate statistical quantification of the input space. This phase starts when the ordering of similar types of neurons is complete. Then tuning is done to let the best neuron be trained most. In this phase, both $\eta$ and the neighborhood size are kept at a constant minimum value.

\subsection{Algorithm}

The algorithm for creation of the SOFM is as follows:

Begin

Initialize maxepoch

For epoch $=1$ to maxepoch

For each input data

Compare the input vector with each neuronal weight vector by determining the Euclidean distance between them. The Euclidean distance ${ }^{\sqrt{*}}$ between the $\mathrm{i}^{\mathrm{in}}$ input data vector ${ }^{*}$ and the $\mathrm{j}^{\mathrm{jin}}$ neuron's weight vector ${ }^{w_{j}}$ is computed using the following formula:

$$
d_{i j}=\sqrt{\sum_{k=1}^{64}\left(x_{i k}-w_{j k}\right)^{2}}
$$

The neuron with the least distance is termed as the winner for that input. Then the winning neuron and the neighboring neurons (the size of the neighborhood depends on the epoch number according to the neighborhood function) are trained according to the following formula, where $\eta$ is the learning constant:

$$
w_{j k}=w_{j k}+\eta \times\left(x_{i k}-w_{j k}\right)
$$

End For

End For

End 


\subsection{Representation of the Map}

After the entire training process is complete, the SOFM is prepared. The SOFM is represented diagrammatically by a $2 \mathrm{D}$ array of circles, each circle representing a neuron. The circles are colored and shaded differently according to whether they are the winner for $E$. coli, Yeast or Mouse.

\subsection{Cluster Centre.}

After mapping all the 108 input data vectors onto the $2 \mathrm{D}$ array of: neurons, it is noted that the inputs from the same species are mapped onto neurons occupying neighboring positions, thus forming different clusters for different species. Now we define the cluster center as the neuron belonging to a particular cluster that has emerged as the winner the maximum number of: times, for that particular species.

\section{SIMULATION RESULTS}

We plot the trained SOFM for $k=6$ below. The neurons that have won for mouse, E. coli, and yeast are shaded differently. The blank circles did not win a single time for any species. We can see that a distinct cluster is formed
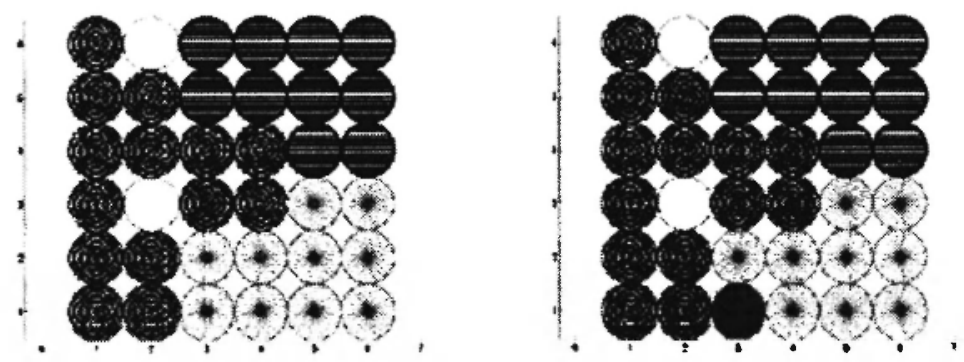

Figure Legend:

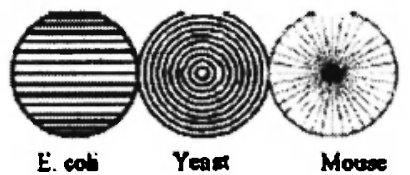

Fig. 3: Trained SOFM showing different clusters and the neuron winning for Mouse 
Now we take a random sequence from the genome sequence of the species Mouse and perform frequency count on it. Using this vector as an input, the distance between this input vector and the weights of the neurons are calculated. The winner for this input, indicated by the filled circle in the above diagram, is found to be a neuron from the map belonging to the cluster for the species Mouse.

The above method offers a scheme for using the SOFM for automatic species identification. Whenever a new sequence is obtained, its DNAdescriptor is computed and the distance between the new input and existing neurons is calculated. The winning neuron will declare to which species it belongs or if it is of a new species then to which phylum the species belongs.

The following figures depict the maps obtained for different values of the map dimension from 5 to 10 . If we chose the map dimension to be less than 4 , the map becomes too small to distinguish between clusters of the 3 species. If it is greater than 11 , we have to increase the number of inputs proportionately, either by increasing the number of species for which the map is constructed or the number of samples per species. They are plotted and compared for optimization of the map dimension.
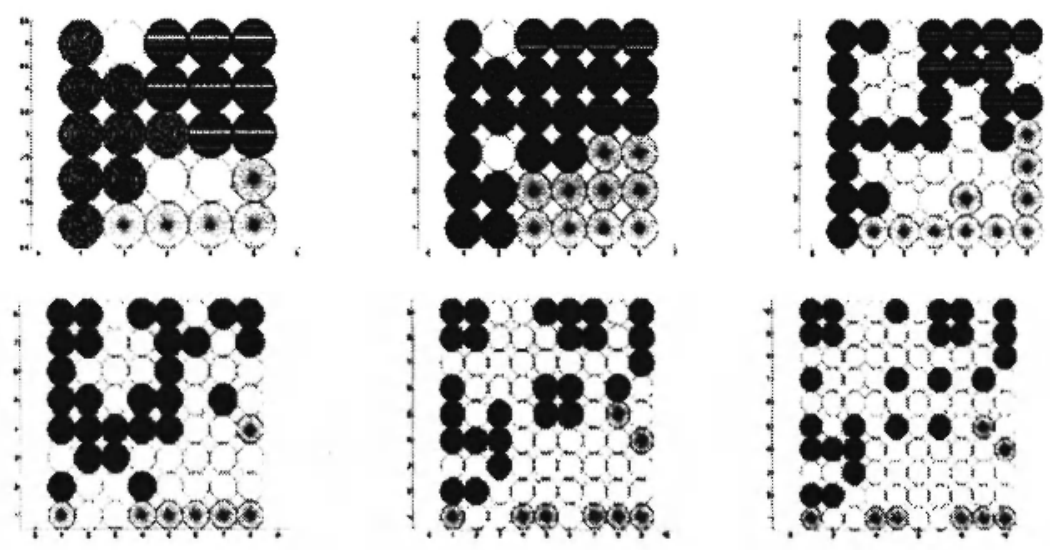

Fig. 4: The SOFMs for map dimension varying from 5 to 10 


\section{PERFORMANCE EVALUATIONS}

We can see that, as the size of the map increases, the cluster corresponding to each species becomes more localized and concentrated. The topological property of a self-organizing feature map may be assessed quantitatively in different ways. One such quantitative measure, called the topographic product (Bauer \& Pawezik, 1992), may be used to compare the faithful behavior of different feature maps pertaining to different dimensionalities. However, the measure is quantitative only when the dimension of the lattice matches that of the input space (Haykin, 1991). Hence, there arose the need to define a new performance index. To estimate how efficient the map is, we first find out the cluster center for each species in each map and then find the Euclidian distance of the other neurons belonging to that cluster from their cluster center. Now the mean and variance of the distance corresponding to each cluster are computed. The following figure contains a plot of the mean distance along with a tolerance margin (depicted by mean \pm variance) for different values of the map dimension for the same species. This parameter i.e. the mean distance of the cluster members from the cluster center is defined as the error and is used as a figure of merit for the SOFM.

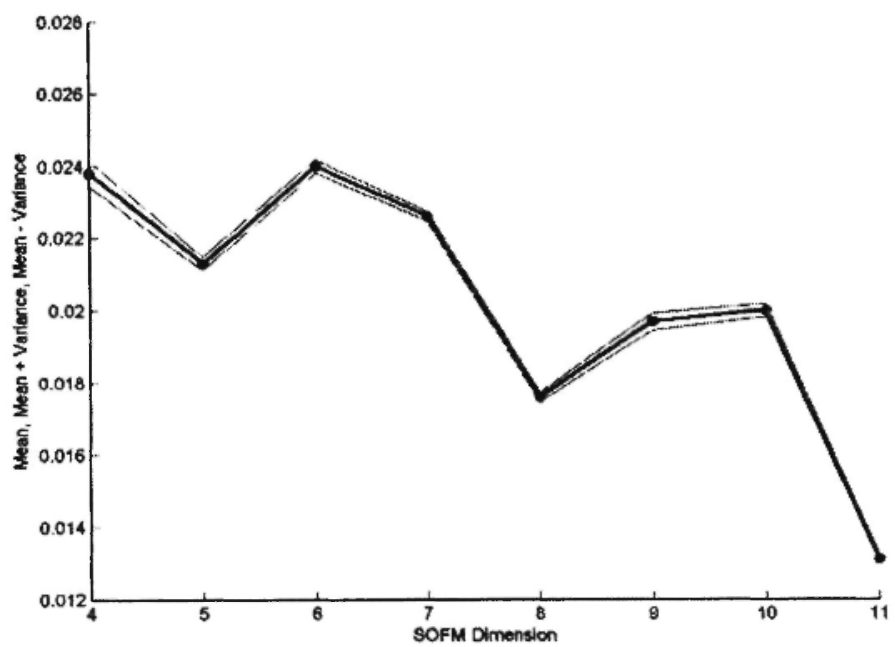

Fig. 5: Error vs. SOFM dimension 
As is clearly visible from the figure, the error decreases as map dimension increases. This signifies that the cluster becomes more concentrated in a smaller region and the neurons that are a part of the cluster emerge as the winner more number of times as the map dimension is increased. This is also validated by the visual representations of the maps shown in Figure 4.

\section{CONCLUSIONS}

Bioinformatics is a new area of science where a combination of statistics, molecular biology, and computational methods is used for analyzing and processing biological information like gene, DNA, RNA, and proteins. However, no significant work has been done toward exploiting the knowledge that the genomic data of a species holds the structural signature of the species, hence can be used for identification and classification of the species. This paper aims to fill this gap.

To our knowledge, this is the first work of its kind to extract information from complete genome sequences and to distinguish between species by feature descriptor diagrams. Since the work entails processing huge amounts of genomic data, the learning ability of neural networks is utilized in this direction.

In this process, we have used PCA to reduce the large dimensions of genome sequence data without loss of accuracy. If only the frequency count is plotted, then we do get some difference from species to species but it is not enough to distinguish between them. This is where PCA comes in. When PCA is applied to the original data we get enough differences between the feature descriptor diagrams of different species that enable us to tell one species from another with the help of these diagrams. Moreover, when feature descriptor diagrams for the same species are calculated, they turned out to be nearly identical, with insignificant variance. Thus, we claim that by constructing feature descriptor diagrams for each species we get an effective identifier for the species. However, we still need a quicker approach for automatic species identification. Here, we utilize the leaning and clustering abilities of computational maps. 
After mapping all the 108 input data vectors onto the $2 \mathrm{D}$ array of neurons, it is noted that data from the same species is mapped onto neurons occupying neighboring positions. Hence, it can be inferred that different vectors computed from different samples of the genomic data of a species are close in many respects and hence are mapped onto neighboring spaces on the map, thus forming separate clusters for different species. It can also be claimed that species that are close in characteristics will have similar DNAdescriptors and hence the clusters corresponding to similar species will lie in neighboring positions. Hence, if clustering techniques are applied to DNAdescriptors of a large number of species, we will see that species that are similar in many respects e.g. Human and Gorilla will be forming sub-clusters within a super-cluster belonging to their families.

Additionally, the SOFM can help us demonstrate homology between new sequences and existing phyla. Whenever a new sequence is obtained, its DNA-descriptor is computed and the distance between the new input and existing neurons is calculated. The winning neuron will declare to which species it belongs or if it is of a new species, then to which phylum the species belongs.

However, the work described in this paper is a pioneer in this regard and carries possibilities for further enhancement in the direction of automatic species identification from genomic data.

\section{REFERENCES}

Adams, M.D., Celniker, S.E., Holt, R.A., Evans, C.A., Gocayne, J.D., Amanatides, P.G., et al 2000. The genome sequence of Drosophila melanogaster, Science, 287, 2185-2195.

Bauer, H.U. and Pawezik, KR. 1992. Quantifying the neighborhood preservation of self-organizing feature maps, IEEE Transactions on Neural Networks, 3, 570-579.

Blattner, F.R., Plunkett, G., Bloch, C.A., Perna, N.T., Burland, V., Riley, M., et al. 1997. The complete genome sequence of Escherichia coli, Science, K-12, 277, 1453-1462.

Cai, L., Juedes, D., and Liakhovitch, E. 2000. Evolutionary computation techniques for multiple sequence alignment, Congress on Evolutionary Computation 2000, 829-835.

Cherry, J.M., Ball, C., Weng, S., Juvik, G., Schimidt, R., Alder, C., et al 1997. Genetic and Physical maps of Saccharomyces cerevisiae, Nature 
(suppl. 6632), 387, 67-73.

Erwin, E., Obermayer, K., and Sculten, K. 1992. II: Self-organizing maps: Ordering, convergence properties and energy functions, Biological Cybernetics, 67, 47-55.

Haykin, S. 1991. Neural Networks: A Comprehensive Foundation, Second Edition, India, Pearson Education, Inc.

Kohonen, T. 1982. Self-organized formation of topologically correct feature maps, Biological Cybernetics, 43, 59-69.

Kohonen, T. Exploration of very large databases by self-organizing feature maps, 1997, International Conference on Neural Networks, vol. I, PL1PL6, Houston, TX, USA.

Needleman, S.B. and Wunsch, C. 1970. A general method applicable to the search for similarities in the amino sequence of two proteins, Journal of Molecular Biology, 48,443-453.

Smith, L.I. 2002. A tutorial on principal components analysis.

Smith, T.F. and Waterman, M.S. 1981. Identification of common molecular subsequences, Journal of Molecular Biology, 147, 195-197.

Waterman, M.S. and Eggert, M. 1987. A new algorithm for best subsequence alignments with applications to tRNA-rRNA, Journal of Molecular Biology, 197, 723-728. 